\title{
THE UPPSALA COMET MAGNITUDE DATABASE
}

\author{
LARS KAMÉL \\ Astronomiska observatoriet, Box 515, S-751 20 Uppsala, Sweden \\ E-mail lars@laban.uu.se.
}

\begin{abstract}
The second part of the Comet Light Curve Catalogue/Atlas (CLICC/A) has been made available by anonymous ftp. This database contains magnitude estimates of periodic comets, reduced to a geocentric distance of $1 \mathrm{AU}$ and corrected for observational effects. The database is described in some detail.
\end{abstract}

\section{Introduction}

CLICC/A is a compilation of magnitude estimates and measurements of periodic comets. The period covered is 1832-1989 for comet P/Encke and 1899-1989 for the other comets. It consists of three parts, two catalogue parts and the atlas. Although only the second part is available by anonymous ftp, all three parts are briefly described in the following sections; more information can be found in the introductions to the catalogue (Kamél, 1991) and the atlas (Kamél, 1992).

\section{The three parts of CLICC/A}

\section{1. THE COMPILED OBSERVATIONS.}

The first part is a catalogue of the compiled observations, including time of observation, observer(s), location, reference(s), telescope and codes describing type of observation, magnitude and telescope. It has been published as a book (Kamél, 1991) and the data are also available on magnetic tape and floppy disks.

\section{2. THE CORRECTED MAGNITUDES.}

The second part is a catalogue of corrected magnitudes, that can be used to obtain light curves of the comets. Corrected means that the magnitudes have been reduced to a geocentric distance of $1 \mathrm{AU}$ and corrected for observational effects, e.g. the delta effect and telescope aperture. These data are available only in digital form. There is one data file per apparition, and the names of the files are based on, but not necessarily the same as, the designations of the apparitions (the exact names depend on the computer system for which the files are intended). The files are text files and their names is the only thing that differs between different computer systems.

\section{3. THE ATLAS.}

The data in the second catalogue part has been used to obtain the light curves that form the atlas. This is available only on paper and has been published by Kamél (1992). 


\section{The availability of the data.}

Access to the data in the second catalogue part is available by anonymous ftp. If you are connected to Internet you type :

ftp ftp.astro.uu.se

When prompted for the username, give anonymous. Identify yourself when prompted for a password. Proceed to the pub/Comets/CLICC directory.

Here you find the MS-DOS version (The reason for this choice is that the MSDOS version can be used on any other computer system, while the opposite is not true) of the second part of CLICC/A. This means that all the data files have suffixes .red. A file name is the designation of the apparition, but with roman numbers converted to arabic, e.g. the data for the apparition 1987 XXXIII (P/Borrelly) are found in the file 198733.red. There is also a file called README and one file called appar.all, containing designations (also those not included in CLICC/A) of apparitions up to 1989 and a file called intro, containing a digital version of the introduction to the catalogue. The data files are available as compressed ZIP-and tar-archives in the files clicc2.zip and clicc2.tar.Z, respectively. Note that these archives should be transferred as binary files.

Readers without access to ftp may order single data files via E-mail from the author. Because of the size of the data set, only small parts of it will be supplied in this way. Those interested in extensive data sets, or the data in the first catalogue part, may order them on floppy disks or magnetic tape from the author.

\section{References}

Kamél, L. : 1991, The Comet Light Curve Catalogue/Atlas. Part I The compiled observations. Uppsala Universitet.

Kamél, L. : 1992, Astron. Astrophys. Suppl. Ser. 92, 85-149. 